Ectopic liver (EL) is a rare congenital abnormality, which is localised most commonly in the wall of the gallbladder. Histoarchitectural abnormalities, which lead to impaired transfer of blood and bile, as well as well demarcation, are characteristic features of ectopic liver nodules. Both features may explain the discrepancies between hepatocellular carcinoma (HCC) cases originating from ectopic liver in comparison to HCC cases originating from orthotopic liver: the strong propensity of ectopic liver to the development of HCC. The latter feature may be linked to the better treatment prognosis in patients with $\mathrm{HCC}$ originating from ectopic liver tissue in comparison to those with HCC within orthotopic liver. In this paper, we discuss these differences based on a unique case of pure HCC, which developed in a small ectopic liver nodule in the pancreas.

Key words: HCC, ectopic liver, hepatocellular carcinoma, liver cancer, pancreas.

Contemp Oncol (Pozn) 2017; 21 (4): 311-314 DOI: https://doi.org/10.5114/wo.2017.72403

\section{Pure hepatocellular carcinoma originates from an ectopic liver nodule located in the pancreas}

\author{
Marcin Braun ${ }^{\star 1,2}$, Wojciech Kuncman ${ }^{\star 1}$, Leszek Teresiński ${ }^{3}$, Piotr Kupnicki ${ }^{4}$, \\ Dorota Jesionek-Kupnicka ${ }^{1}$, Radzislaw Kordek ${ }^{1}$
}

*The first and second author contributed equally to the work.

${ }^{1}$ Department of Pathology, Chair of Oncology, Medical University of Lodz, Poland ${ }^{2}$ Postgraduate School of Molecular Medicine, Medical University of Warsaw, Poland ${ }^{3}$ Department of Pathology, Voivodal Specialistic Hospital, Gorzow Wielkopolski, Poland ${ }^{4}$ Department of Radiology and Diagnostic Imaging, Medical University of Lodz, Poland

\section{Introduction}

Ectopic liver (EL) is defined as liver tissue with no connection to orthotopic liver, while accessory liver is defined as heterotopic liver tissue with a connection to orthotopic liver [1-3]. EL is a rare congenital abnormality with the incidence of $0.23 \%$ reported in the largest autopsy series and $0.47 \%$ reported in the largest laparoscopic series $[1,4,5]$. Gallbladder followed by hepatic ligaments and omentum are the most common localisations of EL; however, cases in other locations throughout the abdomen and thorax have been described $[1,3,4,6]$.

Primary hepatocellular carcinoma ( $\mathrm{HCC})$ is the seventh most common cancer and third most common cause of death from cancer, worldwide [7-9]. HCC typically originates from cirrhotic liver and is related to hepatitis B and C, heavy alcohol use, autoimmune hepatitis, exposure to toxins, or metabolic diseases such as haemochromatosis, $\alpha-1$ antitrypsin deficiency, porphyria, and Wilson's disease [7, 8]. Intriguingly, primary HCC arises in the ectopic liver tissue more commonly in comparison with the normal liver tissue [4, 10]. Furthermore, HCC that develops in EL focus is less commonly associated with risk factors characteristic for HCC arising in orthotopic liver [4, 10]. We describe and discuss a unique case of a 77-year-old male with primary HCC originating from an ectopic liver nodule localised in the pancreas.

\section{Case report}

A 77-year-old male with abdominal discomfort and no significant co-morbidities was subjected to standard diagnostic tests including abdominal ultrasonography (USG). USG scan revealed a heterogenous and slightly hypoechogenic nodule localised in the tail of the pancreas. In a computed tomography scan (CT), the tumour was well delineated and was $25 \mathrm{~mm}$ in diameter (Fig. 1A-D). In the pre-contrast phase, the lesion was slightly hyperdense with a centrally-located hypodense focus of $9 \mathrm{~mm}$ in diameter (Fig. 1A). In the arterial phases, the lesion was hyperdense and enhanced with a $9 \mathrm{~m}$ unenhanced central part, suggesting a necrotic focus (Fig. 1B, C). The lesion was still enhanced in the portal phase (Fig. 1D). The CT image was equivocal and suggested a neuroendocrine tumour of the pancreas (Fig. 1A-D). Additionally, a regular and hypodense mass in the liver was noted. The radiological appearance of this lesion was classic for liver cyst (Fig. 1A-D, white arrow).

The patient underwent complete excision of the tumour. Macroscopically, the tumour was solid and well demarcated. Microscopically, the excision was complete (RO) - the neoplasm was well separated by a thick fibrous band from the adjacent mature pancreatic tissue (Fig. 2A, B). The microscopic 

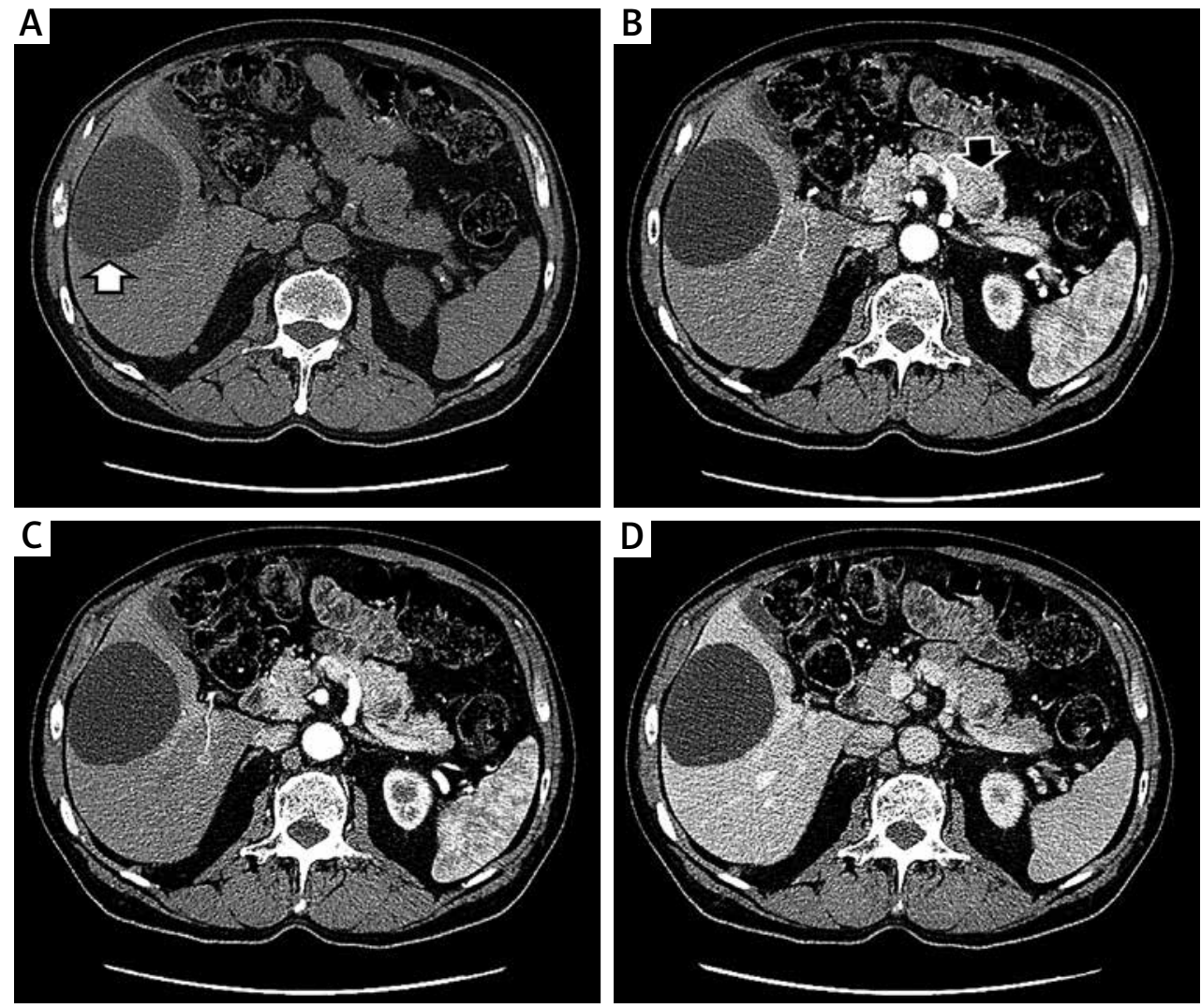

Fig. 1. Computed tomography images of the tumour. The $25-\mathrm{mm}$ tumour in the tail of the pancreas is hyperdense with a 9-mm hypodense central focus. The enhancement of the tumour is seen in the arterial phase and becomes isodense with pancreatic parenchyma in the portal phase. The tumour is indicated by a black arrow. A) Non-enhanced phase. B, C) Arterial phases. D) Portal phase

image showed the neoplastic tissue with scarce areas of residual non-neoplastic tissue. The neoplasm mostly resembled liver; however, the histoarchitecture was irregular, with many sinusoidal vessels between nests of tumour cells (Fig. 2A, B). The tumour cells were pleomorphic, rather large, and highly atypical (Fig. 2B, C). The cytoplasm was abundant with distinct accumulation of bile (Fig. 2C). The nuclei were large, irregular, and with prominent nucleoli (Fig. 2C). No neuroendocrine morphological features were noted (Fig. 2A-C). Additional immunohistochemical stainings were performed. The tumour cells were positive for: cytokeratin 18 (CK18), $\alpha$-fetoprotein (AFP), glypican-3, and hepatocyte and were negative for: cytokeratin 7 (CK7), cytokeratin 20 (CK20), melanosome, chromogranin, synaptophysin, and smooth muscle actin (SMA) (Fig. 2D-F). CK7 was positive in irregular bile ducts, and SMA was positive in the enlarged and abnormal sinusoidal vessels. In the histopathological examination, the tumour was well demarcated and incorporated scarce normal liver tissue. We diagnosed the tumour as primary hepatocellular carcinoma and recommended additional diagnostic tests to exclude another primary focus of the malignancy.

The additional imaging of the abdomen did not show any other abnormalities (apart from a benign liver cyst described above). The AFP level was not elevated. The patient was not infected with hepatitis B or C viruses and did not report alcohol abuse or exposure to toxins. Summing up, there were no signs of any other focus of the malignancy, and we diagnosed the patient with pure primary hepatocellular carcinoma that originated from an ectopic liver tissue localised in the tail of the pancreas. For the next two years, the patient has remained in good condition under close surveillance with un-elevated AFP level and no abnormalities in control CT scans, which further supports our primary diagnosis.

\section{Discussion}

Herein, we discuss an extremely rare presentation of pure hepatocellular carcinoma that originated from an ectopic liver nodule localised in the tail of the pancreas. The case depicts well the clinical, radiological, and pathological differences between HCC that arise in orthotopic versus ectopic liver tissue.

Previously reported cases of ectopic liver were most frequently located in the gallbladder wall, and cases of pancreatic localisation were scarce $[1-3,11]$. According to our knowledge, there were only three reports on primary HCC arising in pancreatic ectopic liver foci $[2,3,11]$. As reported in the literature, the ectopic liver tissue is more prone to develop HCC; however, most frequently such patients do not harbour the typical risk factors for HCC development (HBV/HCV infection, alcohol abuse, exposure to toxins) and the ectopic liver is not cirrhotic $[4,10]$. The phenomenon of carcinogenesis in ectopic liver is attributed to anomalies in ectopic liver tissue architecture, which leads to a worse transfer of blood and bile in ectopic liver [10]. 

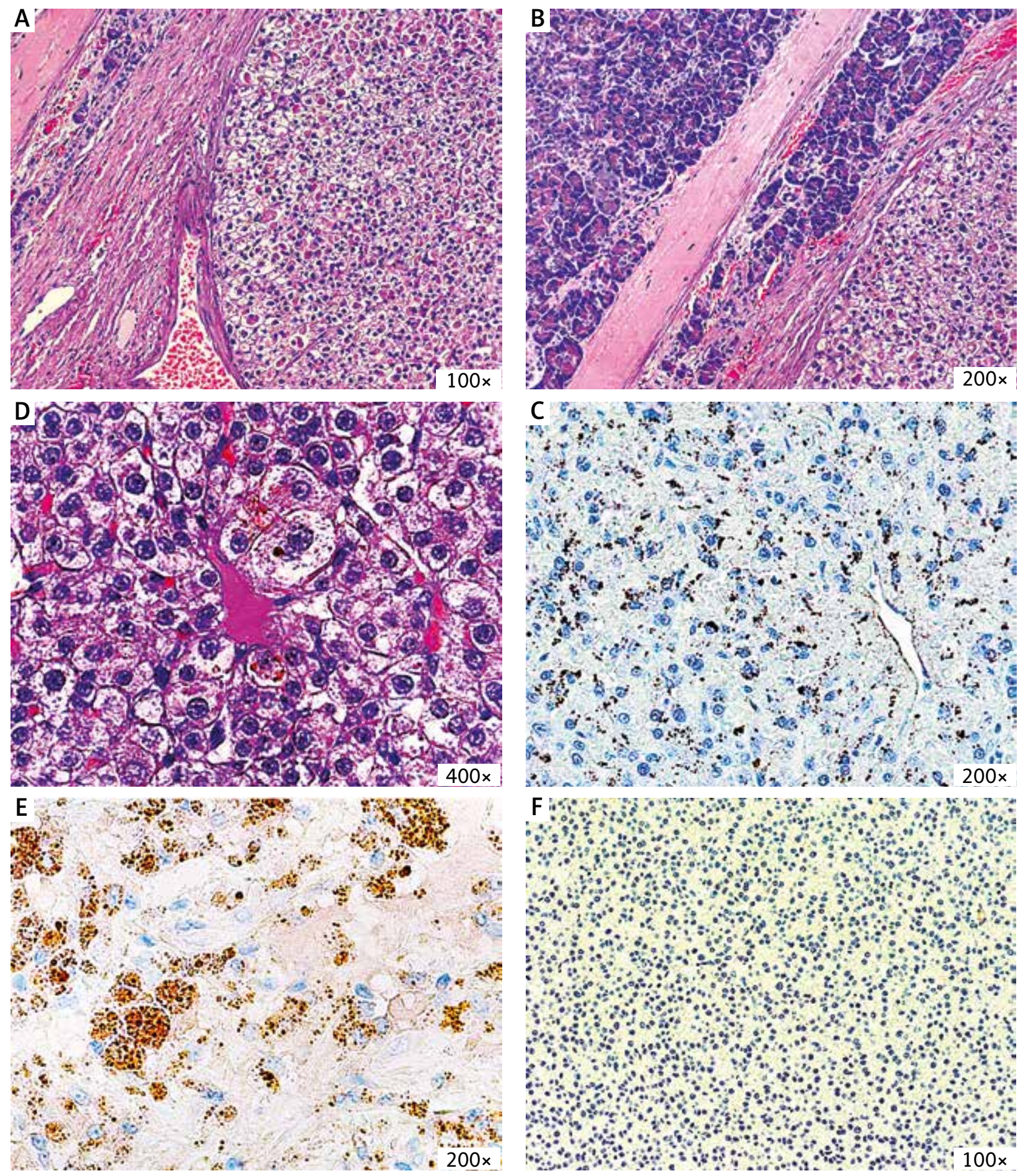

Fig. 2. The histopathological images of the tumour. A-C) In the HE stain the tumour is composed of atypical neoplastic cells that form structures resembling the liver tissue. The accumulation of bile can be seen in C. D, E) The immunohistochemical stains reveal positivity for AFP and glypican-3 and negativity for chromogranin

In our case, the patient did not present with any HCC risk factors, which is a good example of the cited observations.

The radiological appearance of HCC cases in ectopic liver nodules is less characteristic than in the normal liver and may be misleading [4, 12]. The enhancement of the HCC in arterial phases can be less apparent, as well as the rapid "wash-out" effect in the portal phase [4, 12]. These discrepancies were also apparent in our case, where radio- logically the tumour resembled more a pancreatic neuroendocrine tumour than primary or metastatic HCC.

Our histopathological differential diagnosis was inline with literature reports and encompassed pancreatic neuroendocrine tumour and hepatoid carcinoma of the pancreas $[2,3,10]$. The lack of neuroendocrine morphology and negative stainings for chromogranin and synaptophysin excluded the former, while the thick fibrous capsule 
without the connection to both liver and pancreas, as well as the pure HCC morphology, vouched against the latter.

Patients who develop HCCs in ectopic liver foci are most commonly treated successfully with surgical excision, and the prognosis in such cases is excellent $[4,10,11,13]$. This phenomenon may to some extent be explained by a well demarcation and poor vascularity of ectopic liver nodules $[4,10,13]$. The cases of primary HCC in pancreatic foci were cured using surgery with no tumour recurrence, which is similar to our case and most HCC cases developing in ectopic liver [2, 3, 11]. Taken together, such cases (small, single primary HCC developing in ectopic liver foci) should be managed according to guidelines for localised HCC, which indicate either tumour resection or percutaneous ablation therapy followed by active oncological surveillance [14].

The limitation of our case report is the lack of AFP level measurement before the surgery, due to the lack of suspicion of HCC based on the tumour localisation and its equivocal radiological appearance. After the surgery, the AFP was not elevated. The second limitation is the lack of ultrasound imaging of the tumour because the examination was performed in an outpatient clinic and the patient received only the radiological description, which we reported on. The further radiological imaging is well documented.

Our case reminds us that primary hepatocellular carcinoma may originate spontaneously from the ectopic liver tissue in variable organs throughout the abdominal cavity. Secondly, patients with ectopic liver foci, despite being at higher risk of HCC, can be treated more successfully in cases of developing HCC in comparison to patients developing HCC in orthotopic liver.

The authors declare no conflict of interest.

\section{References}

1. Rosai J, Ackerman LV. Rosai and Ackerman's surgical pathology. Mosby, Edinburgh 2011.

2. Kubota K, Kita J, Rokkaku K, et al. Ectopic hepatocellular carcinoma arising from pancreas: a case report and review of the literature. World I Gastroenterol 2007; 13: 4270-3.

3. Steen S, Wolin E, Geller S a, Colquhoun S. Primary hepatocellular carcinoma ("hepatoid" carcinoma) of the pancreas: a case report and review of the literature. Clin Case Rep 2013; 1: 66-71.

4. Arakawa M, Kimura Y, Sakata K et al. Propensity of ectopic liver to hepatocarcinogenesis: case reports and a review of the literature. Hepatology 1999; 29: 57-61.

5. Pulle MV, Mittal T, Dey A, Malik VK. Choristoma (Ectopia) of the liver over gallbladder? A rare congenital anomaly. Curr Med Res Pract 2014; 4: 174-6.

6. Kulkarni RS, Anand AS, Patel AA, Shah SA. Hepatocellular carcino ma presenting as rapidly growing sternal mass: an unusual presentation. J Cancer Metastasis Treat 2016; 41-3.

7. El-Serag HB, Rudolph KL. Hepatocellular carcinoma: epidemiology and molecular carcinogenesis. Gastroenterology 2007; 132: 2557-76.

8. Mittal S, El-Serag HB. Epidemiology of hepatocellular carcinoma: consider the population. J Clin Gastroenterol 2013; 47 Suppl: S2-6.

9. Ferlay J, Soerjomataram I I, Dikshit R, Eser S, Mathers C, Rebelo M, Parkin DM, Forman D, Bray F. Cancer incidence and mortality worldwide: sources, methods and major patterns in GLOBOCAN 2012. Int J Cancer 2014; 136: E359-86.
10. Le Bail B, Carles J, Saric J, Balabaud C, Bioulac-Sage P. Ectopic liver and hepatocarcinogenesis. Hepatology 1999; 30: 585-6.

11. Cardona D, Grobmyer S, Crawford JM, Liu C. Hepatocellular carcinoma arising from ectopic liver tissue in the pancreas. Virchows Arch 2007; 450: 225-9.

12. Kim KA, Park CM, Kim CH, et al. Hepatocellular carcinoma in an ectopic liver: CT findings. Eur Radiol 2003; 13 (suppl. 4): 47-9.

13. Zonca P, Martinek L, Ihnat P. Fleege J. Ectopic liver: Different manifestations, one solution. World J Gastroenterol 2013; 19: 6485-9.

14. Kudo M. Clinical Practice Guidelines for Hepatocellular Carcinoma Differ between Japan, United States, and Europe. Liver Cancer 2015; 4: 85-95.

\section{Address for correspondence}

\section{Marcin Braun}

Department of Pathology

Chair of Oncology

Medical University of Lodz

Pomorska 251

92-213 Lodz, Poland

e-mail: braunmarcin@gmail.com

Submitted: 27.06 .2017

Accepted: 28.07.2017 